PROCEEDINGS OF THE

AMERICAN MATHEMATICAL SOCIETY

Volume 133, Number 12, Pages 3691-3701

S 0002-9939(05)07970-0

Article electronically published on June 3, 2005

\title{
WEAK AXIOMS OF CHOICE FOR METRIC SPACES
}

\author{
KYRIAKOS KEREMEDIS AND ELEFTHERIOS TACHTSIS
}

(Communicated by Carl G. Jockusch, Jr.)

\begin{abstract}
In the framework of ZF, i.e., Zermelo-Fraenkel set theory without the axiom of choice $\mathbf{A C}$, we show that if the family of all non-empty, closed subsets of a metric space $(X, d)$ has a choice function, then so does the family of all non-empty, open subsets of $X$. In addition, we establish that the converse is not provable in $\mathrm{ZF}$.

We also show that the statement "every subspace of the real line $\mathbb{R}$ with the standard topology has a choice function for its family of all closed, non-empty subsets" is equivalent to the weak choice form "every continuum sized family of non-empty subsets of reals has a choice function".
\end{abstract}

\section{Notation AND TERMinOlOGY}

In the following, each of the statements "Form $\mathrm{x}$ " has been considered in [5], where all known implications between these forms are given in Table 1; see http:// www.math.purdue.edu/ jer/Papers/conseq.html.

Definition 1. (1) Let $(X, T)$ be a topological space.

(1a) $X$ is said to be a Loeb space iff the family of all non-empty, closed subsets of $X$ has a choice function.

(1b) $X$ is said to be selective iff the family of all non-empty, open subsets of $X$ has a choice function.

(1c) $X$ is called second countable if it has a countable base for $T$.

(1d) $X$ is called separable if it has a countable dense subset.

(1e) A family $\mathcal{C} \subset(T \backslash\{\emptyset\})$ is called cellular if $\mathcal{C}$ is pairwise disjoint.

(2) Let $(X, d)$ be a metric space.

(2a) A sequence $\left(x_{n}\right)_{n \in \omega}$ of points in $X$ is said to be a Cauchy sequence if $(\forall \epsilon>0)(\exists N \in \omega)(\forall n, m \geq N) d\left(x_{n}, x_{m}\right)<\epsilon$.

(2b) $X$ is called complete or Fréchet complete if every Cauchy sequence in $X$ converges.

(3) $\mathbf{M}(\mathbf{S}, \mathbf{T})$ : Every metric space having the property S, also has the property $\mathrm{T}$.

(4) Form 1, AC: Every family of non-empty sets has a choice function.

(5) Form 8, CAC: AC restricted to countable families.

(6) Form 40, AC(WO): AC restricted to well-ordered families.

Received by the editors May 29, 2004 and, in revised form, August 17, 2004.

2000 Mathematics Subject Classification. Primary 03E25, 54A35, 54D65, 54D70, 54E35, 54E50, 54E99.

Key words and phrases. Axiom of choice, weak axioms of choice, Loeb metric spaces, selective metric spaces, complete metric spaces, separable metric spaces, second countable metric spaces.

(C)2005 American Mathematical Society Reverts to public domain 28 years from publication 3691 
(7) $\mathbf{A} \mathbf{C}_{\text {sel }}(\mathbf{W O})$ : If $(X, d)$ is a selective metric space, then every well-ordered disjoint family of non-empty subsets of $X$ has a choice function.

(8) $\mathbf{A C}_{\mathrm{Loeb}}(\mathbf{W O})$ : If $(X, d)$ is a Loeb metric space, then every well-ordered disjoint family of non-empty subsets of $X$ has a choice function.

(9) $\mathbf{A C}_{\text {Loeb }}(X)$ : If $(X, d)$ is a Loeb metric space, then every family $\mathcal{A}=\left\{A_{i}\right.$ : $i \in I \subseteq X\}$ of non-empty subsets of $X$ has a choice function.

(10) $\mathbf{A C}_{\text {sel }}(X)$ : If $(X, d)$ is a selective metric space, then every family $\mathcal{A}=\left\{A_{i}\right.$ : $i \in I \subseteq X\}$ of non-empty subsets of $X$ has a choice function.

(11) $\mathbf{A C}\left(\aleph^{\omega}\right)$ : Every family $\mathcal{A}=\left\{A_{i}: i \in I \subseteq \aleph^{\omega}\right\}$, $\aleph$ an infinite well-ordered cardinal number 11 of non-empty subsets of $\aleph^{\omega}$ has a choice function.

(12) $\mathbf{A C}_{\aleph^{\omega}}$ : For every $\aleph, \mathrm{AC}\left(\aleph^{\omega}\right)$.

(13) $\mathbf{W O}_{\aleph \omega}$ : For every $\aleph, \aleph^{\omega}$ is well orderable.

(14) Form 79, $\mathbf{A C}(\mathbb{R})$ : AC restricted to families of non-empty subsets of $\mathbb{R}$.

(15) Form 91, PW: The powerset of a well-orderable set is well orderable.

(16) Form 94, CAC $(\mathbb{R})$ : $A C(\mathbb{R})$ restricted to countable families.

(17) Form 212: $\mathrm{AC}(\mathbb{R})$ restricted to continuum sized families.

Proposition 1 ([7]). (ZF) $\left|\omega^{\omega}\right|=|\wp(\omega)|=\left|2^{\omega}\right|=|\mathbb{R}|$.

We shall use the following abbreviations: $\mathbf{2}$ for "second countable", $\mathbf{S}$ for "separable", hS for "hereditarily separable", sel for "selective", hsel for "hereditarily selective" and hLoeb for "hereditarily Loeb".

\section{INTRODUCTION AND SOME KNOWN RESULTS}

The notion of the Loeb space was used implicitly by P. Loeb (see [13]), and was defined explicitly by N. Brunner in [1. Loeb proved, in ZF, that: The Tychonoff product of a well-ordered family of compact topological spaces is compact if it is Loeb.

The notion of the selective space was introduced in 8 , and it was shown, in ZF, that a selective metric space $(X, d)$ has a well-ordered base for its metric topology. Thus, selective metric spaces are always paracompact in ZF (Rudin's proof [15] of Stone's theorem readily implies this fact). For the existence of a non-paracompact metric space $(X, d)$ in $\mathrm{ZF}$, see $[3$.

We show in ZF that a Loeb metric space is selective and that the converse is not provable, i.e., that there exists a ZF model $(M, \in)$ in which there is a metric space $(X, d)$ which is selective but not Loeb.

The key property that makes selective and Loeb metric spaces important is that these spaces are "close" to being well ordered. Namely, they have a well-ordered dense subset (cf. Theorems 3 and $4(\mathrm{i})$ ). Now, if $(X, d)$ is a metric space having a well-ordered dense set $D$, then for every point $x \in X$ one can construct a sequence $\left(d_{n}\right)_{n \in \omega} \subset D$ such that $\lim _{n \rightarrow \infty} d_{n}=x$. Since limits are unique, it follows that $|X| \leq\left|D^{\omega}\right|$. Thus, if the principle $\mathrm{WO}_{\aleph^{\omega}}$ holds true, then $D^{\omega}$ is well orderable, hence $X$ is well orderable, and we may conclude that $\mathrm{M}$ (Loeb,hLoeb) is a theorem of $\mathrm{ZF}+\mathrm{WO}_{\aleph^{\omega}}$. Now, in Fraenkel-Mostowski permutation models for $\mathrm{ZF}^{0}$ set theory (= ZF minus the axiom of regularity), selective and Loeb metric spaces are always well orderable since the axiom $\mathrm{PW}$ (hence the weaker $\mathrm{WO}_{\aleph \omega}$ ) is true in each such model; see [5].

\footnotetext{
${ }^{1}$ Without AC, the cardinal number of a set $X$ is defined (see [16]) as the set of all sets $Y$ of smallest rank for which there exists a 1-1 function $f$ from $Y$ onto $X$.
} 
Before we proceed with the main results let us recall some theorems we shall be needing.

Theorem 1. The following are equivalent:

(i) $\mathrm{CAC}(\mathbb{R})$.

(ii) 4] Every subspace of $\mathbb{R}$ is separable.

(iii) [5], 6] $\mathrm{M}(\mathrm{S}, \mathrm{hS})$.

Theorem 2. (i) 13 (ZF) $\mathbb{R}$ is a Loeb space.

(ii) $8,(Z F) \mathbb{R}$ is a selective space.

(iii) $8 \mathbb{R}$ is hereditarily Loeb implies $\mathrm{CAC}(\mathbb{R})$.

(iv) 8$] \mathbb{R}$ is hereditarily selective iff $\mathrm{CAC}(\mathbb{R})$.

Theorem 3 ([1]). (ZF) A metric space is selective iff it has a well-ordered dense subset.

\section{MAin RESUlts}

Lemma 1. (i) If $(A, \rho)$ is a discrete metric space with well-ordered underlying set, then the Tychonoff product $A^{\omega}$ of $\omega$ copies of A taken with the discrete metric is a Loeb metric space. In particular, the Baire space $\omega^{\omega}$ is Loeb.

(ii) 212 iff $\omega^{\omega}$ is hereditarily Loeb.

(iii) $\mathrm{CAC}(\mathbb{R})$ iff $\omega^{\omega}$ is hereditarily selective.

Proof. (i) Fix $G$ a closed non-empty subset of $X=A^{\omega}$. We describe below, inductively, a way of choosing an element $g$ from $G$. For $i=0$, let $g_{0}$ be the first element of $A$ satisfying $\pi_{0}^{-1}\left(g_{0}\right) \cap G \neq \emptyset$. For $i=k+1$, let $g_{k+1}$ be the first element of $A$ satisfying $\left(\pi_{0}^{-1}\left(g_{0}\right) \cap \pi_{1}^{-1}\left(g_{1}\right) \cap \ldots \cap \pi_{k+1}^{-1}\left(g_{k+1}\right)\right) \cap G \neq \emptyset$. Since $G$ is closed, it follows that $g=\left(g_{i}\right)_{i \in \omega} \in G$ and $X$ is Loeb as required.

(ii) $(\Rightarrow)$ Let $Y$ be a subspace of the Baire space $\mathbb{B}=\omega^{\omega}$. Since $\mathbb{B}$ is second countable, it follows that $Y$ is second countable, hence the family $\mathcal{F}$ of all closed subsets of $Y$ has power $2^{\omega}$. Furthermore, as $\left|\omega^{\omega}\right|=\left|2^{\omega}\right|$ (see Proposition 1), we may apply 212 on $\mathcal{F}$ in order to obtain a choice function on $\mathcal{F}$.

(ii) $(\Leftarrow)$ Since $|\mathbb{B}|=|\mathbb{R}|$ in ZF (by Proposition 1), it suffices to show that every family $\mathcal{A} \subset \wp(\mathbb{B}) \backslash\{\emptyset\}$ such that $|\mathcal{A}|=|\mathbb{B}|$ has a choice function. To this end, fix such a family $\mathcal{A}=\left\{A_{i}: i \in \mathbb{B}\right\}$ of non-empty subsets of $\mathbb{B}$. Since $\mathbb{B}$ is hereditarily Loeb and $\mathbb{B}$ is homeomorphic to $\mathbb{B} \times \mathbb{B}$ (the function $f: \mathbb{B} \longrightarrow \mathbb{B} \times \mathbb{B}$ defined by $f(t)=$ $\left(t^{0}, t^{1}\right)$, where $t^{0}(n)=t(2 n)$ and $t^{1}(n)=t(2 n+1)$ is clearly a homeomorphism), it follows that $\mathbb{B} \times \mathbb{B}$ is also hereditarily Loeb. Put $\mathcal{B}=\left\{A_{i} \times\{i\}: i \in \mathbb{B}\right\}$ and $Y=\bigcup \mathcal{B}$. It can be readily verified that every element of $\mathcal{B}$ is a closed set in the subspace $Y$ of $\mathbb{B} \times \mathbb{B}$. Since $Y$ is a Loeb space, $\mathcal{B}$ has a choice function $f$. Then $g=\left\{\left(i, \pi_{1}(f(i))\right): i \in \mathbb{B}\right\}$, where $\pi_{1}$ is the canonical projection on the first coordinate, is a choice function for $\mathcal{A}$.

(iii) $(\Rightarrow)$ This follows from the facts that $|\mathbb{B}|=|\mathbb{R}|$ and every subspace of $\mathbb{B}$ is second countable (hence separable by $\mathrm{CAC}(\mathbb{R})$ ), and from Theorem 3

(iii) $(\Leftarrow)$ Arguing as in (ii) $(\Leftarrow)$, in order to prove that $\mathrm{CAC}(\mathbb{R})$ holds, it suffices to show that every countable family of pairwise disjoint, non-empty subsets of $\mathbb{B}$ has a choice function. Fix such a family $\mathcal{A}=\left\{A_{i}: i \in \omega\right\}$. Since $\omega$ is homeomorphic to a subspace of $\mathbb{B}$ and $\mathbb{B} \times \mathbb{B}$ is hereditarily selective, it follows that $X=\mathbb{B} \times \omega$ is also hereditarily selective. Thus, the subspace $Y=\bigcup\left\{B_{i}=A_{i} \times\{i\}: i \in \omega\right\}$ of $X$ is selective. Since $\mathcal{B}=\left\{B_{i}: i \in \omega\right\}$ is a family of open subsets of $Y$, it follows 
that $\mathcal{B}$, and consequently $\mathcal{A}$, has a choice function. This completes the proof of the lemma.

Theorem 4. (i) $\mathrm{M}($ Loeb,sel) is a theorem of $Z F$.

(ii) A complete metric space is Loeb iff it is selective.

Proof. (i) Fix $(X, d)$ a Loeb metric space and let $f$ be a choice function on the family $\mathcal{G}$ of all non-empty closed subsets of $X$. In view of Theorem 3 it suffices to show that $X$ has a well-ordered dense subset $G$.

For each $n \in \mathbb{N}$, we construct via a transfinite induction on ordinals a well-ordered set $D_{n} \subset X$ such that $d(x, y) \geq 1 / n$, for all $x, y \in D_{n}, x \neq y$, and $\{D(x, 1 / n)=$ $\left.\{y \in X: d(x, y)<1 / n\}: x \in D_{n}\right\}$ is a cover of $X$.

Fix $n \in \mathbb{N}$. For $i=0$, put $x_{0}=f(X)$. For $i>0$, we first let $U_{i}=X \backslash$ $\bigcup\left\{D\left(x_{j}, 1 / n\right): j<i\right\}$. If $U_{i}=\emptyset$, then we let $D_{n}=\left\{x_{j}: j<i\right\}$ and the induction terminates. Otherwise, we let $x_{i}=f\left(U_{i}\right)$. The induction surely terminates at some ordinal stage.

Clearly $D=\left\{D_{n}: n \in \mathbb{N}\right\}$ is a well-ordered family of well-ordered sets. Thus, $G=\bigcup D$ is a well-ordered set, and it is easy to verify that it is also dense in $X$.

(ii) By (i), every complete, Loeb metric space is selective. Now, let $(X, d)$ be a complete, selective metric space. Then $X$ has a well-ordered dense set $D$, hence a well-ordered base, say $\mathcal{B}=\left\{b_{i}: i \in \aleph\right\}, \aleph$ an infinite well-ordered cardinal. Fix a closed set $F \subset X$. Via a straightforward induction construct a decreasing sequence $\left(c_{n}\right)_{n \in \omega} \subset \mathcal{B}$ such that for all $n \in \omega, \overline{c_{n+1}} \subset c_{n}, \overline{c_{n}} \cap F \neq \emptyset$, and diameter $\left(c_{n}\right)<1 / n$. Using the well ordering of $D$, pick for each $n \in \omega$, the least element $d_{n} \in \overline{c_{n}} \cap D$. Clearly, $\left(d_{n}\right)_{n \in \omega}$ is a Cauchy sequence in $X$, hence it converges to a point $d \in X$. Since $\left\{\overline{c_{n}}: n \in \omega\right\}$ is clearly a neighborhood base of $d$ and $\overline{c_{n}} \cap F \neq \emptyset$ for all $n \in \omega$, it follows that $d \in \bar{F}=F$. We may choose the element $d$ from $F$. Thus, $(X, d)$ is a Loeb metric space as required.

Remark 1. In view of the proof of (i) of Theorem 4 we see that every Loeb metric space $(X, d)$ has a well-ordered base $\mathcal{B}=\bigcup\left\{\mathcal{B}_{n}: n \in \mathbb{N}\right\}$, where for each $n \in \mathbb{N}, \mathcal{B}_{n}$ is a pairwise disjoint family of open discs each one having diameter $1 / n$. Indeed, let $G=\bigcup\left\{D_{n}: n \in \mathbb{N}\right\}$ be the well-ordered dense subset of $X$ constructed in the proof of (i) of Theorem 4 . For each $n \in \mathbb{N}$, let $\mathcal{B}_{n}=\left\{D(x, 1 / 2 n): x \in D_{n}\right\}$. By the construction of $D_{n}$ we have that $\mathcal{B}_{n}$ is a disjoint family for all $n \in \mathbb{N}$. Put $\mathcal{B}=\bigcup\left\{\mathcal{B}_{n}: n \in \mathbb{N}\right\}$. Clearly, $\mathcal{B}$ is well ordered since $|\mathcal{B}|=|G \times \mathbb{N}|$ and $\mathcal{B}$ is a base for the metric topology $T_{d}$ on $X$. Indeed, let $x \in X, \epsilon>0$, and $n \in \mathbb{N}$ such that $1 / n<\epsilon$. By the construction of $D_{2 n}$, we have that $\left\{D(g, 1 / 2 n): g \in D_{2 n}\right\}$ is a cover of $X$, hence $x \in D(g, 1 / 2 n)$ for some $g \in D_{2 n}$. Then $D(g, 1 / 4 n) \in \mathcal{B}_{2 n}$ and $D(g, 1 / 4 n) \subseteq D(x, \epsilon)$.

Theorem 5. $\mathrm{M}($ sel,Loeb $) \rightarrow \mathrm{AC}_{\aleph^{\omega}} \leftrightarrow \mathrm{AC}_{\text {sel }}(X) \leftrightarrow \mathrm{AC}_{\text {Loeb }}(X) \rightarrow 212$.

Proof. $\left(\mathrm{M}(\mathrm{sel}, \mathrm{Loeb}) \rightarrow \mathrm{AC}_{\aleph \omega}\right)$ Let $\aleph$ be an infinite well-ordered cardinal and let $\aleph^{\omega}$ be the Tychonoff product of $\omega$ copies of $\aleph$ taken with the discrete metric. It can be readily verified that $G=\bigcup\left\{G_{n}=\aleph^{n} \times \prod_{i \geq n}\{0\}: n \in \mathbb{N}\right\}$ is a well-ordered dense subset of $\aleph^{\omega}$ of size $\aleph$. Furthermore, $\left|\aleph^{\omega} \backslash G\right|=\left|\aleph^{\omega}\right|$. Indeed, for every $i \in \mathbb{N}$, put $D_{i}=\bigcup\left\{D_{i n}=\aleph^{n} \times \prod_{j \geq n}\{i\}: n \in \mathbb{N}\right\}$. Clearly, $\left|D_{i}\right|=\aleph$ for all $i \in \mathbb{N}$ and $\{G\} \bigcup\left\{D_{i}: i \in \mathbb{N}\right\}$ is a pairwise disjoint family. Define a function $f: \aleph^{\omega} \longrightarrow \aleph^{\omega} \backslash G$ by requiring $f\left(G_{n}\right)=D_{1 n}, f\left(D_{i n}\right)=D_{(i+1) n}$ for all $i, n \in \mathbb{N}$, and $f(x)=x$ for all $x \in \aleph^{\omega} \backslash\left(G \cup\left(\bigcup\left\{D_{i}: i \in \mathbb{N}\right\}\right)\right)$. It is evident that $f$ is an injection, thus 
$\left|\aleph^{\omega}\right| \leq\left|\aleph^{\omega} \backslash G\right|$. Since $\left|\aleph^{\omega} \backslash G\right| \leq\left|\aleph^{\omega}\right|$, it follows from the Cantor-Bernstein theorem (which is provable in ZF; see [7]) that $\left|\aleph^{\omega} \backslash G\right|=\left|\aleph^{\omega}\right|$ as claimed.

Now let $\mathcal{A}=\left\{A_{i}: i \in \aleph^{\omega} \backslash G\right\}$ be a family of non-empty subsets of $\aleph^{\omega}$. Let $Y=(G \times G) \cup\left(\bigcup\left\{\{i\} \times A_{i}: i \in \aleph^{\omega} \backslash G\right\}\right)$ be taken with the topology which inherits as a subspace of $\aleph^{\omega} \times \aleph^{\omega}$. Since $G \times G$ is a well-ordered (e.g. lexicographically) dense subset of $Y$, it follows that $Y$ is selective, and by our hypothesis, $Y$ is Loeb. It is evident that $\{i\} \times A_{i}$ is closed in $Y$ for all $i \in \aleph^{\omega} \backslash G$, hence any choice function on the family $\left\{\{i\} \times A_{i}: i \in \aleph^{\omega} \backslash G\right\}$ immediately yields for a choice function on $\mathcal{A}$, and the proof of the implication is complete.

$\left(\mathrm{AC}_{\aleph^{\omega}} \rightarrow \mathrm{AC}_{\text {sel }}(X)\right)$ If $(X, d)$ is a selective metric space, then $X$ has a wellordered dense subset $D$ of cardinality $\aleph$, for some well-ordered cardinal $\aleph$. Therefore, $|X| \leq\left|\aleph^{\omega}\right|$ and the conclusion readily follows.

$\left(\mathrm{AC}_{\mathrm{sel}}(X) \rightarrow \mathrm{AC}_{\mathrm{Loeb}}(X)\right)$ This follows from Theorem $4(\mathrm{i})$.

$\left(\mathrm{AC}_{\mathrm{Loeb}}(X) \rightarrow \mathrm{AC}_{\aleph \omega}\right)$ This follows immediately from Lemma 1(i).

The last implication follows from the observation that 212 is equivalent to $\mathrm{AC}\left(\aleph_{0}^{\omega}\right)$.

Corollary 1. It is consistent relative to $Z F$ that there exists a selective metric space that is not Loeb.

Proof. In Feferman's forcing model $\mathcal{M} 2$ in [5], 212 fails; see [5]. Thus, by Theorem 5 it follows that in $\mathcal{M} 2$ there exists a selective, non-Loeb metric space. In particular, since $\mathrm{CAC}(\mathbb{R})$ holds in $\mathcal{M} 2$ (see [5]), it follows from Lemma $\mathbb{1}$ (ii), (iii) that the Baire space $\omega^{\omega}$ has a selective, non-Loeb subspace.

Theorem 6. (i) $\mathrm{M}$ (Loeb,hLoeb) iff dense subspaces of complete Loeb metric spaces are Loeb.

(ii) $\mathrm{M}($ Loeb,hLoeb) iff $\mathrm{M}$ (sel,Loeb).

Proof. (i) $(\Rightarrow)$ This is straightforward.

(i) $(\Leftarrow)$ It can be verified in ZF that for every metric space $(X, d)$ there exists an isometric embedding $h$ of $X$ into the complete metric space $Z=B(X, \mathbb{R})$ of all bounded functions $f: X \longrightarrow \mathbb{R}$ under the sup metric $\rho(f, g)=\sup \{|f(x)-g(x)|$ : $x \in X\}$. Thus, $X$ can be densely, isometrically embedded into the complete metric space $\overline{h[X]}$. (To see that $(Z, \rho)$ is complete, let $\left(f_{n}\right)_{n \in \omega}$ be a Cauchy sequence of elements of $Z$. From the definition of $\rho$, it follows that for all $x \in X,\left(f_{n}(x)\right)_{n \in \omega}$ is a Cauchy sequence of reals, hence let $f(x)=\lim _{n \rightarrow \infty} f_{n}(x)$. Clearly, the sequence $\left(f_{n}\right)_{n \in \omega}$ converges uniformly to $f$ on $X$ and consequently $\left(f_{n}\right)_{n \in \omega}$ converges to $f$ in the sense of $\rho$. It is obvious that $f \in Z$, hence $(Z, \rho)$, is complete. For the isometric embedding of $X$ into $Z$ follow the proof of Theorem 1.6, p. 268, in [14.)

Now, fix a Loeb metric space $(X, d)$ and let $Y \subseteq X$. Let $(Z, \rho)$ be the completion of $X$, i.e., $(Z, \rho)$ is complete and $\bar{X}=Z$. (If $f:(X, d) \longrightarrow(B(X, \mathbb{R}), \rho)$ is an isometric embedding, then since $X$ and $f[X]$ are homeomorphic, we may assume that $X \subset \overline{f[X]}=Z$.) By Theorem 4 (i) and Theorem 3, and the fact that $X$ is dense in $Z$, we conclude that $Z$ has a well-ordered dense set, hence $Z$ is selective. Thus, by Theorem 4 (ii), $Z$ is also Loeb and consequently the subspace $\bar{Y}$ of $Z$ is a complete Loeb space. Then by our hypothesis we conclude that $Y$ is also Loeb.

(ii) $(\mathrm{M}($ Loeb,hLoeb) $\rightarrow \mathrm{M}($ sel,Loeb $))$ Fix $(X, d)$ a selective metric space and let $(Z, \rho)$ be the completion of $X$. Since $X$ is dense in $Z$ and $X$ has a well-ordered dense subset, it follows that $Z$ has a well-ordered dense subset, hence $Z$ is selective. 
From Theorem 4 (ii), it follows that $Z$ is also a Loeb space, and by M(Loeb,hLoeb), $X$ is Loeb as required.

$(\mathrm{M}($ sel,Loeb $) \rightarrow \mathrm{M}($ Loeb,hLoeb $))$ By (i), it suffices to show that dense subspaces of complete Loeb metric spaces are Loeb. Let $(X, d)$ be a complete Loeb metric space and let $Y$ be a dense subset of $X$. Since $X$ is complete and Loeb, it follows from Theorem $4(\mathrm{i})$ that $X$ is selective, hence $X$ has a well-ordered dense subset, say of size $\aleph$ for some infinite well-ordered cardinal $\aleph$. Then $|Y| \leq|X| \leq\left|\aleph^{\omega}\right|$ and $Y$ has a well-ordered base $\mathcal{B}=\left\{B_{i}: i \in \aleph\right\}$ for its subspace topology. By Theorem 5 we have that our hypothesis implies $\mathrm{AC}_{\aleph^{\omega} \omega}$, therefore $\mathcal{B}$ has a choice function, say $f$. Then $\left\{f\left(B_{i}\right): i \in \aleph\right\}$ is a well-ordered dense subset of $Y$ which means that $Y$ is selective. By M(sel,Loeb) it follows that $Y$ is Loeb, and the proof is complete.

Theorem 7. (i) $\mathrm{AC}(\mathrm{WO}) \rightarrow \mathrm{AC}_{\text {Loeb }}(\mathrm{WO}) \leftrightarrow \mathrm{AC}_{\text {sel }}(\mathrm{WO}) \leftrightarrow \mathrm{M}($ sel,hsel $)$.

(ii) $\left((\forall X) \mathrm{AC}_{\mathrm{sel}}(X)\right) \rightarrow \mathrm{M}($ sel,hsel $)$.

Proof. (i) $\left(\mathrm{AC}(\mathrm{WO}) \rightarrow \mathrm{AC}_{\mathrm{Loeb}}(\mathrm{WO})\right)$ is evident.

$\left(\mathrm{AC}_{\text {Loeb }}(\mathrm{WO}) \rightarrow \mathrm{AC}_{\mathrm{sel}}(\mathrm{WO})\right)$ Fix $(X, d)$ to be a selective metric space and $\mathcal{A}=\left\{A_{i}: i \in \aleph\right\}$ a well-ordered disjoint family of non-empty subsets of $X$. Let $(Z, \rho)$ be the selective completion of $X$ and without loss of generality (wlog) assume that $X \subset Z$ (see the proof of Theorem 6 (i) $(\Leftarrow)$ ). By Theorem 4 (ii) we have that $Z$ is a Loeb space, hence by $\mathrm{AC}_{\text {Loeb }}(\mathrm{WO})$ it follows that $\mathcal{A}$ has a choice function.

$\left(\mathrm{AC}_{\text {sel }}(\mathrm{WO}) \rightarrow \mathrm{M}(\mathrm{sel}, \mathrm{hsel})\right)$ Fix $(X, d)$ to be a selective metric space and $Y \subset X$. Then $Y$ has a well-ordered base $\mathcal{B}$ (wlog assume that $\mathcal{B}$ is disjoint; notice that $X \times \aleph$ is selective) and since $\mathcal{B} \subset \wp(X)$, it follows from $\mathrm{AC}_{\text {sel }}$ (WO) that any choice function on $\mathcal{B}$ generates a well-ordered dense subset of $Y$. Thus, $Y$ is selective as required.

$\left(\mathrm{M}(\right.$ sel,hsel $\left.) \rightarrow \mathrm{AC}_{\text {Loeb }}(\mathrm{WO})\right)$ Fix $\mathcal{A}=\left\{A_{i}: i \in \aleph\right\}$, where $\aleph$ is an infinite well-ordered cardinal number, a disjoint family of non-empty subsets of a Loeb metric space $(Y, \rho)$, where $\rho$ is bounded by 1 . Clearly, for each $i \in \aleph$, the metric space $\left(Z_{i}=Y \times\{i\}, \rho_{i}\right)$, where $\rho_{i}((x, i),(y, i))=\rho(x, y)$, has a definable choice function for the family of all of its non-empty, closed subsets and by Theorem 4 (i) for the family of all of its non-empty, open subsets. Let $\left(X=\bigcup\left\{Z_{i}: i \in \aleph\right\}, \sigma\right)$, $\sigma(x, y)=\rho_{i}(x, y)$ if $x, y \in Z_{i}$, and $\sigma(x, y)=1$ otherwise. Clearly, $\sigma$ is a metric on $X$ producing the disjoint union topology on $X$. It is evident that $X$ is a selective metric space. Without loss of generality we assume that for every $i \in I, A_{i} \subset Z_{i}$. By M(sel, hsel), it follows that the subspace $Z=\bigcup \mathcal{A}$ of $X$ is selective, hence $Z$ has a well-ordered dense subset, say $G$. On the basis of $G$ and the fact that each $A_{i}$ is an open subset of $Z$ we can easily define a choice function for the family $\mathcal{A}$.

(ii) $\left((\forall X) \mathrm{AC}_{\text {sel }}(X)\right) \rightarrow \mathrm{M}($ sel,hsel $)$. This can be proved as in $\left(\mathrm{AC}_{\text {sel }}(\mathrm{WO}) \rightarrow\right.$ $\mathrm{M}($ sel,hsel $))$ noticing now that a subspace of a selective metric space $(X, d)$ has a well-ordered base of size at most $|X|$.

Theorem 8. If $(X, d)$ is a Loeb metric space having a well-ordered dense set $D$ such that $|\wp(D)| \leq|X|$, then $\mathrm{AC}_{\text {Loeb }}(X)$ implies that $X$ is hereditarily Loeb. In particular, 212 implies that $\mathbb{R}$ is hereditarily Loeb.

Proof. Let $(X, d)$ be a Loeb metric space and let $Y \subset X$. Let $\mathcal{G}$ be the family of all non-empty closed subsets of $Y$. It is easy to verify that $|\mathcal{G}| \leq|X|(X$ has a well-ordered base $B$ of size $|D|$, hence $B_{Y}=\{b \cap Y: b \in B\}$ is a well-ordered base for $Y$ of size $|D|$. Thus, the subspace topology on $Y$ has size at most $|\wp(D)| \leq|X|$ (the function which maps every open set $O$ in $Y$ to $\left\{A \in B_{Y}: A \subset O\right\}$ is obviously 


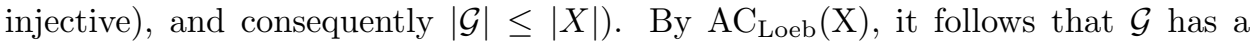
choice function and $Y$ is Loeb as required.

In 8 ] it has been shown that " $\mathbb{R}$ is hereditarily Loeb implies $\mathrm{CAC}(\mathbb{R})$ " and it was asked whether the reverse implication holds. The answer (in the negative) to this question was our main motivation for the study of this paper, and it will come as the conclusion of the following two theorems.

Theorem 9. Let $(X, d)$ be a complete, Loeb metric space and let $\kappa$ be an infinite, well-ordered, regular cardinal number. If every non-empty open subset of $X$ has a cellular family of size at least $\kappa$, then $\kappa^{\omega}$ homeomorphically embeds in $X$. In particular, the Baire space $\omega^{\omega}$ homeomorphically embeds in $\mathbb{R}$.

Proof. From the proof of (i) of Theorem $4, X$ has a well-ordered dense subset $D=\bigcup\left\{D_{n}: n \in \mathbb{N}\right\}$, where for each $n \in \mathbb{N}, d(x, y) \geq 1 / n$ for all $x, y \in D_{n}$ with $x \neq y$. Let $\aleph$ be the cardinality of $D$. From Remark 1 , the family $\mathcal{B}=$ $\bigcup\left\{\mathcal{B}_{n}=\left\{D(x, 1 / 2 n): x \in D_{n}\right\}: n \in \mathbb{N}\right\}$ is a well-ordered base for $X$ of size $\aleph$ and let $\mathcal{B}=\left\{b_{j}: j \in \aleph\right\}$. From our hypothesis on the open subsets of $X$, it follows that $\kappa \leq \aleph$. To see this, let $b$ be any element in $\mathcal{B}$. Then $b$ has a cellular family $\left\{c_{i}: i \in I\right\}$ of open subsets in $b$ (hence in $X$ ) such that $\kappa \leq|I|$. For each $i \in I$, and using the well ordering of $\mathcal{B}$, pick an element $d_{i} \in \mathcal{B}$ such that $d_{i} \subseteq c_{i}$ for all $i \in I$. It follows that

$$
\kappa \leq|I|=\left|\left\{d_{i}: i \in I\right\}\right| \leq|\mathcal{B}|=\aleph .
$$

We now consider the following two cases:

Case 1: $\kappa>\aleph_{0}$. By induction we construct an upside down $\omega$-tree $L=\bigcup\left\{L_{n}\right.$ : $n \in \omega\}$ such that $L$ is uniformly well ordered of size at least $\kappa, L_{n}$ consists of closed balls for all $n \in \omega,\left|L_{n}\right| \geq \kappa$, and for each $t \in L_{n}$ there exist at least $\kappa$ elements $s \in L_{n+1}$ such that $s \subseteq t$.

For $n=0$ we first note that there exists an $m \in \mathbb{N}$ such that $\kappa \leq\left|\mathcal{B}_{m}\right|$. If not, first let $\mathcal{C} \subseteq \mathcal{B}$ such that $|\mathcal{C}|=\kappa$. Then $\kappa=|\mathcal{C}|=\left|\bigcup\left\{\mathcal{C}_{n}=\mathcal{C} \cap \mathcal{B}_{n}: n \in \mathbb{N}\right\}\right|$ and since $\left|\mathcal{C}_{n}\right|<\kappa$, it follows that $\kappa$ is singular (not regular), a contradiction. Let $n_{0}$ be the least $n \in \mathbb{N}$ such that $\kappa \leq\left|\mathcal{B}_{n}\right|$ and put $L_{0}=\left\{\overline{D\left(x, 1 / 4 n_{0}\right)}: x \in D_{n_{0}}\right\}$.

Suppose that we have constructed the level $L_{n}=\left\{l_{n i}: i \in\left|L_{n}\right|\right\}$ for some $n>0$.

By transfinite induction on $\left|L_{n}\right|$ we construct $L_{n+1}$ as follows. For $i=0$ and each $m \in \mathbb{N}$, let $C_{m}=\left\{b \in \mathcal{B}_{m}: b \subseteq l_{n 0}\right\}$. Then there must be an $m \in \mathbb{N}$ such that $\kappa \leq\left|C_{m}\right|$. If not, let $m \in \mathbb{N}$ such that $C_{m} \neq \emptyset$ and $b \in C_{m}$. Then $b$ has a cellular family $F$, for which without loss of generality we may assume to be a subset of $\mathcal{B}$, having size at least $\kappa$. Then $\kappa \leq|F| \leq\left|\bigcup\left\{C_{m}: m \in \mathbb{N}\right\}\right|$ and since each $\left|C_{m}\right|<\kappa$, it follows that $\kappa$ is singular. This is a contradiction. Let $m_{0}$ be the first $m \in \mathbb{N}$ such that $\kappa \leq\left|C_{m}\right|$. For $i>0$ we may work similarly to the case $i=0$ and find a family $C_{m_{i}}=\left\{b \in \mathcal{B}_{m_{i}}: b \subseteq l_{n i}\right\}$ with $\kappa \leq\left|C_{m_{i}}\right|$. Finally, put $L_{n+1}=\left\{\overline{D\left(x, 1 / 4 m_{i}\right)}: x \in D_{m_{i}}, D\left(x, 1 / 2 m_{i}\right) \in C_{m_{i}}, i \in\left|L_{n}\right|\right\}$.

Let $L=\bigcup\left\{L_{n}: n \in \mathbb{N}\right\}$. It is clear that $L$ is a well-ordered set (being a well-ordered union of sets each having a fixed well ordering) of size, say $\lambda$, with $\kappa \leq \lambda \leq \aleph$. If $P$ is a path through $L$, then since for every $F \in P, F$ is closed, $F \cap D \neq \emptyset, \lim _{A \in P} \operatorname{diameter}(A)=0$, and $X$ is complete, it follows that $\cap P$ is a singleton, say $\left\{y_{P}\right\}$. Now, for every $f \in \kappa^{\omega}$, and since each node of $L$ spans in at least $\kappa$ descendants, we may pick a path $P_{f}=\left(p_{f(i)}\right)_{i<\kappa}$ so that $f$ corresponds to $y_{P_{f}}$. Moreover, as each $L_{n}$ is an antichain, it follows that if $f, g \in \kappa^{\omega}, f \neq g$, then $P_{f} \neq P_{g}$ and consequently $y_{P_{f}} \neq y_{P_{g}}$. Therefore, the function $h$ which maps every 
element $f \in \kappa^{\omega}$ to $y_{P_{f}}$ is an injection onto $Y=h\left(\kappa^{\omega}\right)$. Now, let $f \in \kappa^{\omega}$ and $O=$ $\pi_{i_{1}}^{-1}\left(f\left(i_{1}\right)\right) \cap \ldots \cap \pi_{i_{n}}^{-1}\left(f\left(i_{n}\right)\right)$ be a basic neighborhood of $f$. Without loss of generality assume that $i_{j}=j$ for all $j, j=1, \ldots, n$. Then $h(O)=\left(p_{f(n)}\right)^{\circ} \cap Y$, hence $h$ maps basic open sets of $\kappa^{\omega}$ to open sets of $Y$. Thus, $h$ is open and similarly continuous, hence a homeomorphism. This means that $\kappa^{\omega}$ homeomorphically embeds in $X$ as required.

Case 2: $\kappa=\aleph_{0}$. By induction we construct an upside down $\omega$-tree $L=\bigcup\left\{L_{n}\right.$ : $n \in \omega\}$ such that $L$ is uniformly well ordered of size $\aleph_{0}, L_{n}$ consists of closed balls for all $n \in \omega,\left|L_{n}\right|=\aleph_{0}$, and for each $t \in L_{n}$ there exist $\aleph_{0}$ many elements $s \in L_{n+1}$ such that $s \subseteq t$.

For $n=0$ we construct the level $L_{0}$ by induction as follows: For $i=0$, let $j_{0}=\min \left\{j \in \aleph: \overline{b_{j}} \neq X\right\}$, where $b_{j}, j \in \aleph$, are the elements of the base $\mathcal{B}$ (see the opening paragraph of the proof). $j_{0}$ is definable. To see this, let $b$ be any element of $\mathcal{B}$ with a cellular family $F=\left\{f_{i}: i \in I\right\},|I| \geq \aleph_{0}$. Since $\mathcal{B}$ is well ordered, we may assume without loss of generality that $F \subseteq \mathcal{B}$. It is evident that $\overline{f_{i}} \neq X$ for all $i \in I$. Put $c_{00}=\overline{b_{j_{0}}}$. For $i \in \mathbb{N}$ and having constructed pairwise disjoint closed sets $c_{0 j}, j<i$, such that $\left(c_{0 j}\right)^{\circ} \in \mathcal{B}$ for all $j<i$ and $X \neq z_{i}=\bigcup\left\{c_{0 j}: j<i\right\}$, we first let $j_{i}=\min \left\{j \in \aleph: \overline{b_{j}} \subseteq\left(X \backslash z_{i}\right)\right.$ and $\left.X \neq \overline{b_{j}} \cup z_{i}\right\}$. $j_{i}$ is definable. Indeed, since $X \neq z_{i}$, there exists $b \in \mathcal{B}$ such that $\bar{b} \subseteq\left(X \backslash z_{i}\right)$. Let $F$ be an infinite cellular family for $b$ such that $F \subseteq \mathcal{B}$. Let $f, g \in F, f \neq g$. Then $\bar{f} \cup \bar{g} \subseteq\left(X \backslash z_{i}\right)$ and $g \cap\left(\bar{f} \cup z_{i}\right)=\emptyset$ meaning that $X \neq \bar{f} \cup z_{i}$. Put $c_{0 i}=\overline{b_{j_{i}}}$. Let $L_{0}=\left\{c_{0 i}: i \in \omega\right\}$.

Suppose that we have constructed the level $L_{n}=\left\{l_{n i}: i \in \omega\right\}$ for some $n>0$.

By induction we construct $L_{n+1}$ as follows: For each $i \in \omega$ we work exactly as in the case $n=0$, but now using $l_{n i}$ instead of $X$ and obtain an infinite, pairwise disjoint family $C_{l_{n i}}$ of closed subsets of $l_{n i}$ whose interiors belong to $\mathcal{B}$. Put $L_{n+1}=$ $\bigcup\left\{C_{l_{n i}}: i \in \omega\right\}$.

Put $L=\bigcup\left\{L_{n}: n \in \omega\right\}$. We may continue now as in the last paragraph of the case $\kappa>\aleph_{0}$ in order to verify that $\aleph_{0}^{\omega}$ homeomorphically embeds in $X$.

The assertion that the Baire space $\omega^{\omega}$ homeomorphically embeds in $\mathbb{R}$ follows from the fact that $\mathbb{R}$ with the standard metric is a complete, Loeb metric space, $\aleph_{0}$ is a regular cardinal number and each open set of $\mathbb{R}$ has a (unique) decomposition in $\aleph_{0}$ open subsets.

Theorem 10. The following are equivalent:

(i) Form 212.

(ii) $\mathrm{M}(2, \mathrm{hLoeb})$.

(iii) $\mathrm{M}(2, \mathrm{Loeb})$.

(iv) $\mathrm{M}(\mathrm{S}, \mathrm{hLoeb})$.

(v) $\mathrm{M}(\mathrm{S}, \mathrm{Loeb})$.

(vi) $\mathbb{R}$ is hereditarily Loeb.

Proof. (i) $\rightarrow$ (ii) Let $(X, d)$ be a second countable metric space and let $Y \subset X$. Clearly, the metric space $\left(Y, d_{Y}\right)$, where $d_{Y}$ denotes the restriction of the metric $d$ on $Y \times Y$, is also second countable. Let $\mathcal{B}=\left\{B_{n}: n \in \omega\right\}$ be a base for the metric topology $T_{d_{Y}}$ on $Y$. Then the function $f: T_{d_{Y}} \longrightarrow \wp(\omega)$ defined by $f(O)=\left\{n \in \omega: B_{n} \subset O\right\}$ for all $O \in T_{d_{Y}}$ is 1-1. Furthermore, since $\left(Y, d_{Y}\right)$ is a $\mathrm{T}_{2}$ space, it can be readily verified that the function $g: Y \longrightarrow \wp(\omega)$ defined by $g(y)=\left\{n \in \omega: y \in B_{n}\right\}$ for all $y \in Y$ is 1-1. Thus, $|Y| \leq|\wp(\omega)|$ and $\left|T_{d_{Y}}\right| \leq|\wp(\omega)|$ and since $|\wp(\omega)|=|\mathbb{R}|$ (see Proposition 1), it follows that $|Y| \leq|\mathbb{R}|$ and $\left|T_{d_{Y}}\right| \leq|\mathbb{R}|$. 
Therefore, $|\mathcal{F}| \leq|\mathbb{R}|$, where $\mathcal{F}$ is the family of all non-empty, closed subsets of $Y$, and the elements of $\mathcal{F}$ can be viewed as subsets of $\mathbb{R}$. By 212 there exists a choice function $f$ for the family $\mathcal{F}$. Thus, $Y$ is a Loeb space and $X$ is hereditarily Loeb as required.

(ii) $\rightarrow$ (iii) $\rightarrow$ (iv) $\rightarrow$ (v) are straightforward.

(v) $\rightarrow$ (vi) Let $Y \subset \mathbb{R}$ with the subspace topology. Since $\mathbb{R}$ is hereditarily second countable, it follows that the family $\mathcal{C}$ of all non-empty closed subsets of $Y$ has size at most $|\mathbb{R}|=\left|\mathbb{Q}^{c}\right|$, so let $\mathcal{C}=\left\{C_{i}: i \in I \subset \mathbb{Q}^{c}\right\}$. Consider the subspace $Z=(\mathbb{Q} \times \mathbb{Q}) \cup\left(\bigcup\left\{D_{i}=\{i\} \times C_{i}: i \in I\right\}\right)$ of $\mathbb{R} \times \mathbb{R}$. Clearly, $Z$ is a separable metric space, thus by (v), $Z$ is Loeb. Since each $D_{i}$ is closed in $Z$, any choice function on $D=\left\{D_{i}: i \in I\right\}$ yields a choice function on $\mathcal{C}$, and $Y$ is Loeb as required.

(vi) $\rightarrow$ (i) By Theorem 9 we have that the Baire space $\omega^{\omega}$ homeomorphically embeds in $\mathbb{R}$, so by our hypothesis, $\omega^{\omega}$ is hereditarily Loeb. Then by Lemma 1(ii), 212 holds as required.

Remark 2. We leave as an easy exercise for the reader the verification that the following statements can be added to the list of Theorem $10, \mathbb{R}^{n}$ is hereditarily Loeb for all $n \in \mathbb{N}, \mathbb{R}^{\omega}$ is hereditarily Loeb, and $2^{\omega}$ is hereditarily Loeb.

\section{INDEPENDENCE RESULTS}

Theorem 11. In $Z F, \mathrm{CAC}(\mathbb{R})$ does not imply $\mathbb{R}$ is hereditarily Loeb.

Proof. In Feferman's forcing model $\mathcal{M} 2$ in $[5, \operatorname{CAC}(\mathbb{R})$ holds whereas 212 fails; see [5]. From Theorem 10 we conclude that in $\mathcal{M} 2, \mathbb{R}$ has a subspace which is not Loeb.

Theorem 12. (i) In $Z F^{0}$, neither $\mathrm{M}($ Loeb,hLoeb) nor $\mathrm{M}$ (sel,hsel) imply $\mathrm{AC}(\mathrm{WO})$.

(ii) In $Z F, \mathrm{M}(\mathrm{sel}, \mathrm{hsel})$ is strictly weaker than $\mathrm{AC}_{\aleph^{\omega}}$, hence strictly weaker than $\mathrm{M}($ Loeb,hLoeb).

Proof. (i) First we point out that the statements M(Loeb,hLoeb) and M(sel,hsel) hold true in every permutation model, since any Loeb (or selective) metric space is well orderable in such a model; see the Introduction. To establish our independence result, we first recall the description of the permutation model we constructed in [10. The set of atoms $A=\bigcup\left\{A_{n}: n \in \omega\right\}$, where $A_{n}=\left\{a_{n, x}: x \in \mathbb{R}\right\}$ and $A_{n}$ is ordered like the reals by $\leq_{n}$. Thus, $\left(A_{n}, \leq_{n}\right)$ is order isomorphic to $(\mathbb{R}, \leq)$ for all $n \in \omega . \mathcal{G}$ is the group of all permutations $\pi$ on $A$ such that $\left.\pi\right|_{A_{n}} \in \operatorname{Aut}\left(A_{n}, \leq_{n}\right)$ for all $n \in \omega$, where $\operatorname{Aut}\left(A_{n}, \leq_{n}\right)$ is the group of all order automorphisms on $A_{n}$. The normal ideal $I$ of supports is the ideal generated by the set of all finite unions $\bigcup_{i \leq n} A_{i}, n \in \omega$. Let $\mathcal{N}$ be the resulting permutation model.

We show now that $\mathrm{AC}(\mathrm{WO})$ is false in $\mathcal{N}$ by establishing that the family $\mathcal{A}=$ $\left\{A_{n}: n \in \omega\right\}$ does not have a choice function in $\mathcal{N} . \mathcal{A} \in \mathcal{N}$ and it is countable in $\mathcal{N}$ since both $\mathcal{A}$ and its enumeration $f=\left\{\left(n, A_{n}\right): n \in \omega\right\}$ have empty support, i.e., every permutation $\pi \in \mathcal{G}$ fixes $\mathcal{A}$ and $f$. Assume on the contrary that $\mathcal{A}$ has a choice function $f \in \mathcal{N}$ with support $E$, that is, $\operatorname{fix}(E) \subseteq \operatorname{sym}(f)$, where $\operatorname{fix}(E)=\{\pi \in \mathcal{G}:(\forall a \in E) \pi(a)=a\}$ and $\operatorname{sym}(f)=\{\pi \in \mathcal{G}: \pi(f)=f\}$. Suppose that $E=A_{0} \cup A_{1} \cup \ldots \cup A_{k}$ for some $k \in \omega$ and that $f\left(A_{k+1}\right)=a_{k+1, x}$ for some $x \in \mathbb{R}$. Define the permutation $\phi$ on $A$ by requiring $\left.\phi\right|_{A_{n}}$ be the identity for all $n \neq k+1$, and $\phi\left(a_{k+1, z}\right)=a_{k+1, z+1}$, for all $z \in \mathbb{R}$. It is evident that $\phi \in \mathcal{G}$ since $\left.\phi\right|_{A_{k+1}}$ is a translation on $A_{k+1}$ and the identity mapping on $A \backslash A_{k+1}$, and 
that $\phi$ fixes $E$ pointwise. Hence, $\phi(f)=f$ and we have $\left(A_{k+1}, a_{k+1, x}\right) \in f \rightarrow$ $\left(\phi\left(A_{k+1}\right), \phi\left(a_{k+1, x}\right)\right) \in \phi(f) \rightarrow\left(A_{k+1}, a_{k+1, x+1}\right) \in f$. This is a contradiction, hence $\mathcal{A}$ admits no choice function in the model $\mathcal{N}$.

(ii) In Feferman's forcing model, model $\mathcal{M} 2$ in [5], $\mathrm{AC}(\mathrm{WO})$ holds (see [5]), hence by Theorem 7(i), M(sel,hsel) also holds in this model. However, 212 fails in $\mathcal{M} 2$ (see [5] and [17), and consequently by Theorems [5, and 6(ii), $\mathrm{AC}_{\aleph \omega}$ and $\mathrm{M}($ Loeb,hLoeb) also fail in that model.

Theorem 13. (i) In ZF, $\mathrm{WO}_{\aleph \omega}$, hence $\mathrm{M}(\mathrm{Loeb}, \mathrm{hLoeb})$, is strictly weaker than AC.

(ii) In ZF, 212 is strictly weaker than $\mathrm{WO}_{\aleph \omega}$.

Proof. (i) In [9, Theorem 11] we constructed a symmetric extension model $(\mathcal{N}, \in)$ of a countable transitive model $(\mathcal{M}, \in)$ of $\mathrm{ZF}+(V=L)$ using an $\aleph_{1}$-closed partially ordered set $\mathbb{P}$ of forcing conditions. Due to $\mathbb{P}$, all cardinals of $\mathcal{M}$ are preserved in $\mathcal{N}$ and for every $\aleph \in \mathcal{M}$, no new functions $f: \omega \longrightarrow \aleph$ are added; see [12, p. 214, Theorem 6.14]. Therefore, $\left(\aleph^{\omega}\right)^{\mathcal{M}}=\left(\aleph^{\omega}\right)^{\mathcal{N}}$ and since $\mathrm{AC}$ is true of $\mathcal{M}$, $\aleph^{\omega}$ is wellorderable in $\mathcal{N}$ for every well-ordered cardinal $\aleph$. Thus, $\mathrm{WO}_{\aleph^{\omega}}$ is valid in $\mathcal{N}$, and consequently $\mathrm{M}$ (Loeb,hLoeb) is also true of $\mathcal{N}$ (see the Introduction). However, the principle $\mathrm{PW}$ fails in $\mathcal{N}$, since $\mathbb{R}$ is well orderable in $\mathcal{N}$ whereas $\wp(\mathbb{R})$ is not; see 9 . The conclusion now follows from the fact that in $\mathrm{ZF}, \mathrm{AC}$ is equivalent to PW; see [2].

(ii) Clearly, $\mathrm{WO}_{\aleph \omega}$ implies that $\omega^{\omega}$ is well orderable, hence $\mathbb{R}$ is well orderable. Thus, $\mathrm{WO}_{\aleph \omega}$ implies the axiom $\mathrm{AC}(\mathbb{R})$. It is well known that 212 does not imply $\mathrm{AC}(\mathbb{R})$ in $\mathrm{ZF}$ (see [5]), and the independence result follows.

\section{REFERENCES}

[1] N. Brunner, Products of compact spaces in the least permutation model, Zeitschr. F. Math. Logic und Grundlagen d. Math. 31 (1985), 441-448. MR0808771 (87h:03077)

[2] U. Felgner and T. Jech, Variants of the axiom of choice in set theory with atoms, Fund. Math. 79 (1973), 79-85. MR0319760 (47:8302)

[3] C. Good, I. J. Tree, S. Watson, On Stone's theorem and the axiom of choice, Proc. Amer. Math. Soc. 126 (1998), 1211-1218. MR1425122 (98f:03044)

[4] H. Herrlich and G. E. Strecker, When is $\mathbb{N}$ Lindelöf?, Comment. Math. Univ. Carolinae 38 (1998), 553-556. MR1485075 (99c:03070)

[5] P. Howard and J. E. Rubin, Consequences of the Axiom of Choice, Amer. Math. Soc., Math. Surveys and Monographs, Vol. 59, Providence (RI), 1998. MR1637107(99h:03026)

[6] T. Jech, The Axiom of Choice, North-Holland, Amsterdam, 1973. MR0396271 (53:139)

[7] T. Jech, Set theory, Academic Press, New-York, 1978. MR0506523 (80a:03062)

[8] K. Keremedis, Consequences of the Failure of the Axiom of Choice in the Theory of Lindelöf Metric Spaces, Math. Log. Quart. 50, No 2, (2004), 141-151. MR2037733 (2005b:54051)

[9] K. Keremedis and E. Tachtsis, Some weak forms of the axiom of choice restricted to the real line, Math. Log. Quart. 47 (2001), 413-422. MR.1847457 (2002e:03077)

[10] K. Keremedis and E. Tachtsis, Countable Sums and Products of Metrizable Spaces in ZF, Math. Log. Quart. 51, No. 1, (2005), 95-103.

[11] K. Keremedis and E. Tachtsis, Topology in the absence of the axiom of choice, Scientiae Mathematicae Japonicae 59, No. 2 (2004), 357-406. MR2062202

[12] K. Kunen, Set Theory. An Introduction to Independence Proofs, North-Holland, Amsterdam, 1980. MR0597342 (82f:03001)

[13] P. A. Loeb, A new proof of the Tychonoff theorem, Am. Math. Monthly 72 (1965), 711-717. MR0190896 (32:8306)

[14] J. R. Munkres, Topology, Prentice-Hall, Inc., New Jersey, 1975. MR0464128 (57:4063)

[15] M. E. Rudin, A new proof that metric spaces are paracompact, Proc. Amer. Math. Soc. 20 (1969), 603. MR0236876 (38:5170) 
[16] D. S. Scott, Definitions by abstraction in axiomatic set theory, Bull. Amer. Math. Soc. 61 (1955), 442.

[17] J. Truss, The axiom of choice for linearly ordered families, Fund. Math. 99 (1978), 133-139. MR0480029 (58:228)

[18] S. Willard, General Topology, Addison-Wesley Publ. Co., 1970. MR0264581(41:9173)

Department of Mathematics, University of the Aegean, Karlovassi 83200, Samos, Greece

E-mail address: kker@aegean.gr

Department of Statistics and Actuarial Science, University of the Aegean, KarloVAssi 83200, Samos, Greece

E-mail address: 1tah@aegean.gr 